\title{
A Modeling Method of JPEG Quantization Table for QVGA Images
}

\author{
G.-M. Jeong, J.-D. Lee, S.-I. Choi, D.-W. Kang
}

\section{Gu-Min Jeong}

School of Electrical Engineering

Kookmin University, Seoul, Korea

E-mail: gm1004@kookmin.ac.kr

\author{
Jong-Duck Lee \\ Avionics Center \\ LIG Nex1, Daejeong, Korea \\ E-mail: jdlee81@lignex1.com
}

\section{Sang-Il Choi}

School of Electrical Engineering and Computer Science

Seoul National University, Seoul, Korea

E-mail: kara@csl.snu.ac.kr

\section{Dong-Wook Kang \\ Corresponding author \\ School of Electrical Engineering \\ Kookmin University, Seoul, Korea \\ E-mail: dwkang@kookmin.ac.kr}

\begin{abstract}
This paper presents a new JPEG quantization table design method for mobile phone images. Although the screen size of mobile phones is very small, the full information of the image should nevertheless be represented. Moreover, the high frequency components of the mobile phone images may contain important information. In order to enhance the performance of mobile JPEG images, these high frequency components should be compensated using an appropriate quantization table. Considering these characteristics, we propose a modeling method of the quantization table for compensating the high frequency components of the mobile images while sacrificing their low frequency components. We select the optimized pre-emphasis factor and bias factor using various sets of $240 \times 320$ images and show that the proposed method improves the performance in terms of size and PSNR.

Keywords: JPEG, Quantization Table, Mobile QVGA Image, Frequency Compen-
\end{abstract} sation

\section{Introduction}

In still image coding, JPEG [1] [2] has become a de facto standard and shows good performance for digital pictures. In the case of mobile phone images, JPEG is also widely used. Nowadays, although VGA $(480 \times 640)$ or SVGA $(600 \times 800)$ screens are already used in smartphones, QVGA $(240 \times 320)$ LCDs, which are very small compared to those used in PCs or digital cameras, are generally adopted in handsets. For these reasons, the handset images may have different characteristics from those of PCs or digital cameras [3].

In mobile phone images, the whole information must be described within a small image size. When comparing small and large size images of the same scene, the frequency characteristics in the $8 \times 8$ blocks can be different from each other. That is, the effect of the high frequency components can be increased compared to that of the low frequency components in small size images. Therefore, in handset images, 
especially QVGA images, the high frequency components can be relatively more important than in PC or digital camera images, due to the differences in the image size. Considering these characteristics, in order to improve the image quality and compression ratio for mobile images, it is possible to design a specific quantization table by decreasing the quantization values for the high frequency components and increasing those for the low frequency components.

In this paper, we propose a new JPEG quantization table design for $240 \times 320$ mobile images extending the results in [4]. We present a new modeling scheme of the quantization table by considering the characteristics of mobile images and optimize the pre-emphasis factor and the bias factor using the $240 \times 320$ images which are serviced by a telecommunication company [5]. Especially, in contrast to R-D optimization [6], we model the quantization table by making full use of the standard quantization table. There is no need to send the quantization table, as in the case of R-D optimization. Since only the pre-emphasis factor and the bias factor are needed in the proposed method, it can be simply applied to the JPEG encoder/decoder. The simulation results show that the proposed method works well.

The remainder of this paper is organized as follows. In Section 2, the characteristics of mobile images are conceptually discussed. In Sections 3 and 4, we present the proposed quantization table modeling scheme and pre-emphasis factor optimization using tests, respectively. The conclusions are presented in Section 5.

\section{The characteristics of handset images}

Recently, with the support of $3 \mathrm{G}$ wireless communication which provides a high speed data rate, there is a growing demand to increase the size of the screen in order for the user to enjoy various multimedia contents. However, to guarantee the portability and mobility of mobile phones, the extent to increase the screen size of mobile phones is limited.

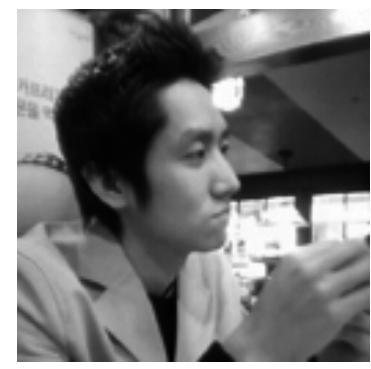

(a)

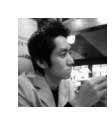

(b)

Figure 1: Sample image and $1 / 4 \times 1 / 4$ image

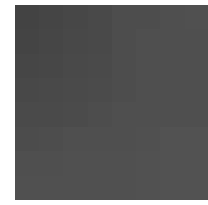

(a)

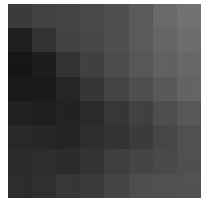

(b)

Figure 2: The right-lower blocks of the images in Fig. 1(a) and Fig. 1(b)

The small size of the mobile phone causes the frequency characteristics of mobile images to differ from those of PC or digital camera images. For example, let us consider the images shown in Fig. 1. Fig. 1(a) and Fig. 1(b) are $512 \times 512$ and $128 \times 128$ images, respectively. Fig. 2(a) and Fig. 2(b) are the 
right-lower $8 \times 8$ blocks in those images in Fig. 1. As seen in Fig. 2, the right-lower $8 \times 8$ block in Fig. 2(b) has more high frequency components than that in Fig. 2(a).

As can be seen in Fig. 1 and Fig. 2, we can think conceptually that the importance of the high frequency components increases in the $8 \times 8$ blocks for the small size images.

Likewise, to design the JPEG quantization table for mobile QVGA images, the high frequency components should be compensated. However, compensating the high frequency components may increase the compressed file size. For these reasons, we propose a quantization table modeling scheme for compensating the high frequency components while sacrificing the low frequency components in order not to increase the compressed file size. In this way, we hope to achieve a better PSNR and bpp than those obtained using the standard table for QVGA images.

\section{Quantization table modeling for mobile images}

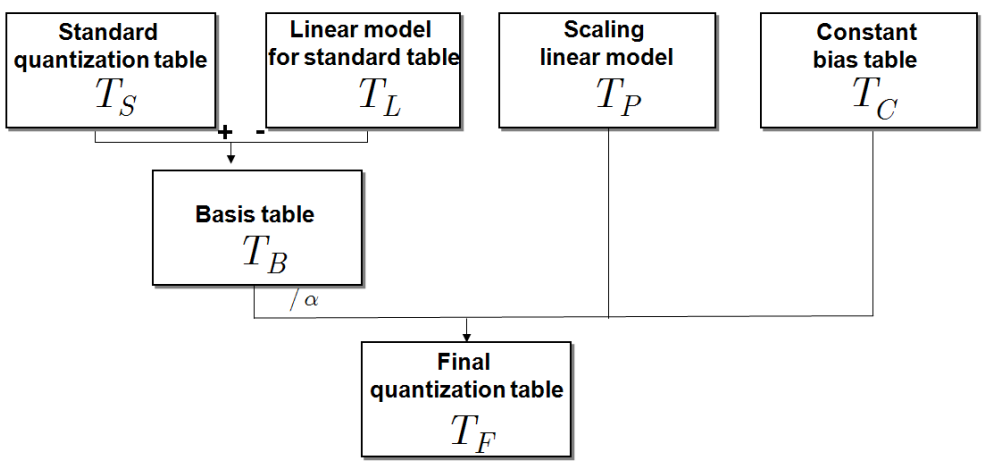

Figure 3: The proposed modeling scheme of quantization table

We propose a new modeling method for the JPEG quantization table used for mobile images which compensates the high frequency components. Fig. 3 shows the overall design of the proposed method. To make full use of the standard quantization table, we obtain the final quantization table $T_{F}$ from the standard quantization table $T_{S}$, pre-emphasis factor $\alpha$ and bias factor $\beta$.

First, the linear model of the standard table $T_{L}$ is derived from $T_{S}$. We obtain the basis table $T_{B}$ by subtracting $T_{L}$ from $T_{S}$. Next, the scaling linear model $T_{P}$ and the constant bias table $T_{C}$ are calculated from the pre-emphasis factor $\alpha$ and the bias factor $\beta$ respectively. As a result, the final quantization table $T_{F}$ is derived using $T_{F}=T_{P}+T_{B} / \alpha+T_{C}$.

Let us describe the proposed modeling method in more detail. We set $T_{L}(1,1)=T_{S}(1,1)$ and $T_{L}(8,8)=T_{S}(8,8)$. Based on $T_{L}(1,1)$ and $T_{L}(8,8)$, we can obtain $T_{L}$ using (1). Using $T_{S}$ and $T_{L}, T_{B}$ is calculated as $T_{B}=T_{S}-T_{L}$. Note that (1) is also used for the calculation of $T_{P}$.

$$
\begin{array}{ll}
T(x, x) \equiv T(x)=\frac{T(8)-T(1)}{7}(x-1)+T(1), \text { if } x=y \\
T(x, y)=T\left(\frac{x+y}{2}\right), & \text { if } x+y \text { is even } \\
T(x, y)=\frac{T\left(\frac{x+y-1}{2}\right)+T\left(\frac{x+y+1}{2}\right)}{2}, & \text { if } x+y \text { is odd }
\end{array}
$$

where $T(x, y)$ means the $(x, y)$ component of the $8 \times 8$ matrix table $T$.

In the proposed modeling, we compensate for the high frequency components by increasing the low frequency components of the table and decreasing the high frequency components of the table. Therefore, 
we set $T_{P}(1,1)$ and $T_{P}(8,8)$ according to the pre-emphasis factor $\alpha$ as follows:

$$
T_{P}(1,1)=\alpha T_{L}(1,1), \quad T_{P}(8,8)=\frac{1}{\alpha} T_{L}(8,8) .
$$

$T_{P}$ is also a linear model and can be calculated from $T_{P}(1,1)$ and $T_{P}(8,8)$ using (1). Also $T_{C}$ is a constant bias table using bias factor $\beta$, which is obtained by $T_{C}=\beta I$.

We can obtain the final quantization table $T_{F}$ as follows:

$$
T_{F}=T_{P}+T_{B} / \alpha+T_{C}=T_{P}+\left(T_{S}-T_{L}\right) / \alpha+T_{C} .
$$

As $\alpha$ increases, the effect of the high frequency components increases, while that of the low frequency components decreases. Also, the detailed bias is adjusted using $\beta$.

\begin{tabular}{|c|c|c|c|c|c|c|c|c|c|c|c|c|c|c|c|c|c|c|c|c|c|c|c|c|c|c|c|c|c|c|}
\hline 16 & 11 & & 16 & 24 & 40 & 51 & 61 & 16 & 21 & 27 & 33 & 39 & 45 & 51 & 57 & 32 & 33 & 34 & 35 & 37 & 38 & \begin{tabular}{l|l}
39 & 40
\end{tabular} & 32 & 28 & 25 & 26 & 29 & 35 & 39 & 42 \\
\hline 12 & 12 & 14 & 19 & 26 & 58 & 60 & 55 & 21 & 27 & 33 & 39 & 45 & 51 & 57 & 63 & 33 & 34 & 35 & 37 & 38 & $33 \mid$ & \begin{tabular}{l|l|}
40 & 42 \\
\end{tabular} & 28 & 26 & 25 & 2 & 28 & 42 & 41 & \\
\hline 14 & 13 & 16 & 24 & 40 & 57 & 69 & 56 & 27 & 33 & 39 & 45 & 5 & 57 & 63 & 69 & 34 & 35 & 37 & 38 & 39 & 40 & \begin{tabular}{l|l}
42 & 43 \\
\end{tabular} & 27 & 25 & 25 & 27 & 33 & 40 & 45 & 36 \\
\hline 14 & \begin{tabular}{|l|}
17 \\
\end{tabular} & 22 & 29 & 51 & 87 & 80 & 6 & 33 & 39 & 45 & 51 & 5 & 6 & 69 & 75 & 35 & 37 & 38 & 39 & 40 & 42 & \begin{tabular}{l|l}
43 & 44 \\
\end{tabular} & 25 & 26 & 26 & 28 & $37 \mid$ & 54 & 48 & 37 \\
\hline 18 & 22 & 3 & 56 & 68 & $\begin{array}{l}109 \\
\end{array}$ & 10 & 7 & 36 & 4. & 51 & 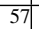 & 6 & 6 & 75 & 81 & \begin{tabular}{|l|}
37 \\
\end{tabular} & 38 & $x$ & 40 & 42 & 43 & 44 & 26 & 26 & 32 & 39 & 44 & 63 & 58 & 43 \\
\hline 24 & 35 & 55 & 64 & 81 & 104 & 113 & 92 & 45 & 51 & 57 & \begin{tabular}{l|l}
63 \\
\end{tabular} & 6 & 75 & 81 & 8 & 38 & 39 & 40 & 42 & 43 & 44 & \begin{tabular}{l|l}
45 & 47 \\
\end{tabular} & 27 & 31 & 39 & 42 & 49 & 58 & 61 & 49 \\
\hline 49 & 64 & 78 & 87 & 103 & 121 & 120 & 101 & 51 & 57 & 63 & 69 & 75 & 81 & 87 & 93 & 39 & 40 & 42 & 43 & 44 & 45 & 47 & 38 & 43 & 49 & 52 & 58 & 65 & 63 & 52 \\
\hline 7 & 92 & & 98 & 112 & 100 & 103 & 99 & 57 & 63 & 69 & 75 & 81 & 87 & 93 & 99 & 40 & 42 & 43 & 444 & $4 \mathrm{~S}$ & 47 & 48 & 47 & 56 & 56 & 55 & 60 & 53 & 53 & \\
\hline
\end{tabular}

Table 1: $T_{S}, T_{L}, T_{P}$ and $T_{F}$ when $\alpha=2, \beta=0$

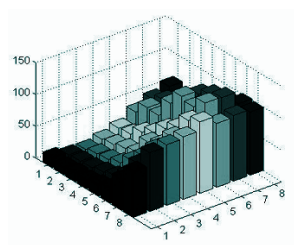

(a) $T_{S}$

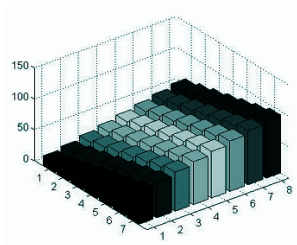

(b) $T_{L}$

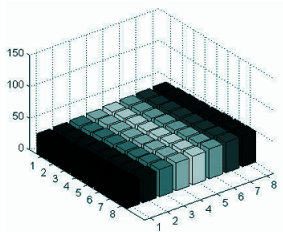

(c) $T_{P}$

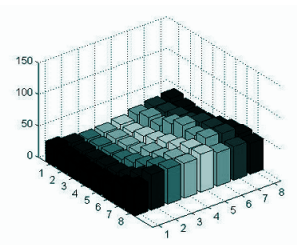

(d) $T_{F}$

Figure 4: $T_{S}, T_{L}, T_{P}$ and $T_{F}$ when $\alpha=2, \beta=0$

Table 1 and Fig. 4 show $T_{S}, T_{L}, T_{P}$ and $T_{F}$ when $\alpha=2$ and $\beta=0$, respectively. As shown in $T_{F}$, the low frequency values are increased and the high frequency values are decreased in the quantization table.

\section{Experiments for $240 \times 320$ handset images}

To obtain the quantization table for QVGA images,we selected 300 images from the SK Telecom photo service site [5], which consist of 100 facial images, 100 whole body images and 100 background images. Fig. 5 shows sample pictures among the test images.

Based on the proposed method, quantization tables are selected in three ways, which are optimizing a performance index for $\alpha$, optimizing that performance index for both $\alpha$ and $\beta$, and selecting a quantization table in order to reduce the size of compressed images preserving image quality.

Next, to validate the selected pre-emphasis factors and bias factors, we choose another 300 images, as shown in Fig. 6 and adopt these selected $\alpha$ 's and $\beta$ 's to these examples.

\subsection{Pre-emphasis factor optimization for $240 \times 320$ handset images [4]}

We choose the optimum pre-emphasis factor $\alpha$ to minimize the Lagrangian cost given by $J(\alpha)=$ $D(\alpha)+\lambda R(\alpha)$, where $D(\alpha)$ and $R(\alpha)$ are the distortion of the reconstructed images and the rate required 


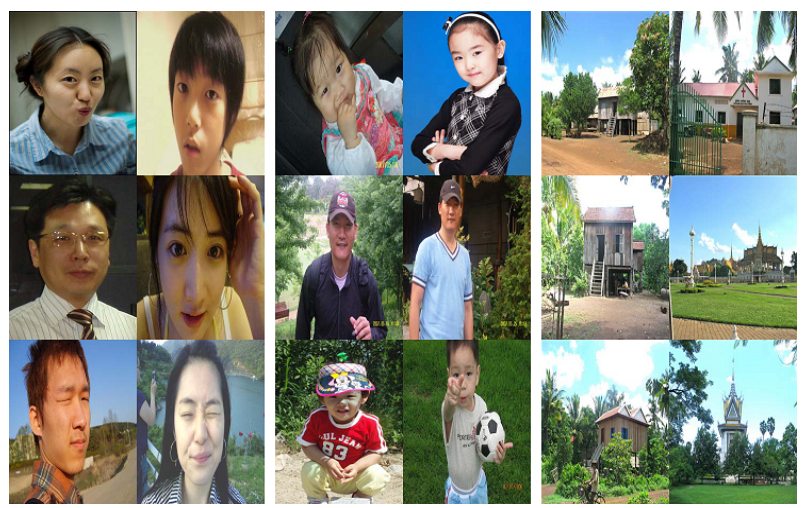

Figure 5: Samples of test images to select quantization tables
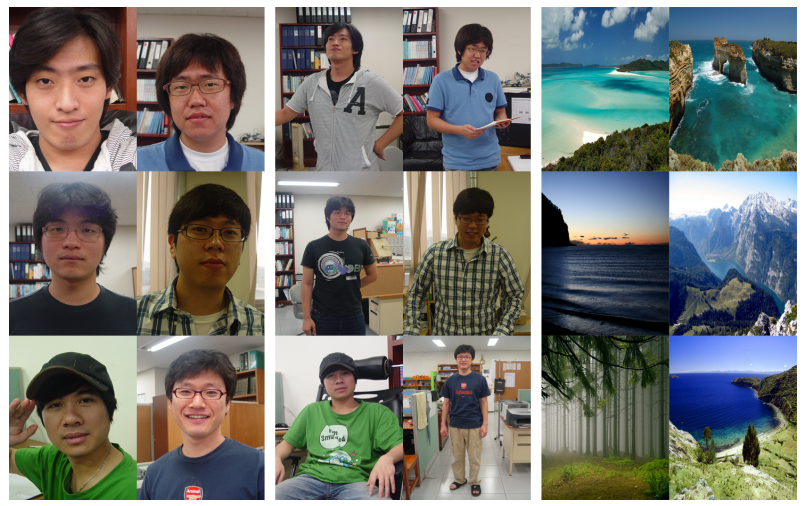

Figure 6: Samples of test images to validate the selected quantization tables

to encode the test images with the corresponding quantization table, respectively, and $\lambda$ is the Lagrangian multiplier. An exhaustive search for the optimum value of $\lambda$ concludes that the Lagrangian cost tends to be minimized at $\lambda=1.125$. Here, we set $\beta=0$.

The cost values are optimal for 1.6, 1.7 and 1.9 for the facial, body and background images, respectively, and we select these values. (Also, as shown in Table 2, if we do not divide the images into these three categories, without any loss of generality, we can use $\alpha=1.9$.)

\subsection{Pre-emphasis factor and bias factor optimization for $240 \times 320$ handset images}

We choose the optimum pre-emphasis factor $\alpha$ and bias factor $\beta$ to minimize the Lagrangian cost given by $J(\alpha, \beta)=D(\alpha, \beta)+\lambda R(\alpha, \beta)$ with respect to the same $\lambda=1.125$ as in Section 4.1. Table 3 shows the selected pre-emphasis factors and bias factors considering the cost function. Comparing to the result in Table 2, there is a little improvement in the cost values.

\subsection{Selection of quantization table considering image size preserving image quality}

Also, we select $\alpha$ and $\beta$ in order to minimize the image size preserving image quality. Table 4 shows the selected pre-emphasis factors and bias factors. 
Table 2: Cost values $J(\alpha)$ for variable $\alpha(\beta=0)$

\begin{tabular}{|c|c|c|c|c|}
\hline$\alpha$ & Face & Body & Background & total \\
\hline 1.4 & 20.31 & 44.50 & 38.35 & 103.16 \\
\hline 1.5 & 20.32 & 44.47 & 38.07 & 102.86 \\
\hline 1.6 & $\mathbf{2 0 . 1 6}$ & 44.19 & 37.52 & 101.87 \\
\hline 1.7 & 20.21 & $\mathbf{4 4 . 1 6}$ & 37.25 & 101.62 \\
\hline 1.8 & 20.29 & 44.24 & 37.19 & 101.72 \\
\hline 1.9 & 20.28 & 44.24 & $\mathbf{3 6 . 9 3}$ & $\mathbf{1 0 1 . 4 5}$ \\
\hline 2.0 & 20.56 & 44.40 & 37.19 & 102.15 \\
\hline 2.1 & 20.72 & 44.59 & 37.22 & 102.53 \\
\hline
\end{tabular}

Table 3: Cost values $J(\alpha, \beta)$ for selected $\alpha$ 's and $\beta$ 's

\begin{tabular}{|c|c|c|c|}
\hline & $\alpha$ & $\beta$ & Cost value \\
\hline Face & 2.3 & -4 & 20.12 \\
\hline Body & 2.1 & -1 & 44.06 \\
\hline Background & 2.3 & -1 & 36.77 \\
\hline
\end{tabular}

\subsection{Experimental results}

Let us denote the selected quantization tables in Section 4.1, Section 4.2 and Section 4.3 as $T_{F 1}, T_{F_{2}}$ and $T_{F 3}$, respectively.

Table 5 shows the performance improvement using the proposed method for the images in Fig. 5. For $T_{F 2}$, there are improvements of $7.58 \%, 7.93 \%$ and $2.85 \%$ in the size and $0.18 \mathrm{~dB}, 0.16 \mathrm{~dB}$ and $0.53 \mathrm{~dB}$ in the PSNR, for the face, body and background images, respectively. Also, for $T_{F 3}$, there are improvements of $10.23 \%, 11.47 \%$ and $9.45 \%$ in the size and $0.03 \mathrm{~dB}, 0.01 \mathrm{~dB}$ and $0.18 \mathrm{~dB}$ in the PSNR, respectively.

Next, to validate the selected pre-emphasis factors and bias factors, we apply the selected factors to other 300 images as shown in Fig. 6. Table 6 shows the performance improvement using the proposed method for the images in Fig. 6. For $T_{F_{2}}$, there are improvements of $8.51 \%, 6.16 \%$ and $7.14 \%$ in the size and $0.22 \mathrm{~dB}, 0.05 \mathrm{~dB}$ and $0.19 \mathrm{~dB}$ in the PSNR, for the face, body and background images, respectively. Also, for $T_{F_{3}}$, there are improvements of $11.03 \%, 9.77 \%$ and $13.13 \%$ in the size and $0.08 \mathrm{~dB}, 0.2 \mathrm{~dB}$ and $0.02 \mathrm{~dB}$ in the PSNR, respectively. 
Table 4: Selected $\alpha$ 's and $\beta$ 's

\begin{tabular}{|c|c|c|}
\hline & $\alpha$ & $\beta$ \\
\hline Face & 2.1 & -1 \\
\hline Body & 2.3 & 1 \\
\hline Background & 2.1 & 4 \\
\hline
\end{tabular}

Table 5: Performance improvement using the proposed method compared to the standard quantization table

\begin{tabular}{|c|c|c|c|c|c|c|}
\hline & \multicolumn{2}{|c|}{ Face } & \multicolumn{2}{c|}{ Body } & \multicolumn{2}{c|}{ Background } \\
\cline { 2 - 7 } & $\mathrm{bpp}$ & $\mathrm{dB}$ & $\mathrm{bpp}$ & $\mathrm{dB}$ & bpp & $\mathrm{dB}$ \\
\hline$T_{S}$ & 1.192 & 35.32 & 1.520 & 31.71 & 1.576 & 32.18 \\
\hline$T_{F_{1}}$ & 1.120 & 35.40 & 1.424 & 31.86 & 1.543 & 32.69 \\
\hline Improvements & $6.04 \%$ & $0.08 \mathrm{~dB}$ & $6.31 \%$ & $0.15 \mathrm{~dB}$ & $2.09 \%$ & $0.51 \mathrm{~dB}$ \\
\hline$T_{F_{2}}$ & 1.107 & 35.50 & 1.407 & 31.87 & 1.532 & 32.71 \\
\hline Improvements & $7.58 \%$ & $0.18 \mathrm{~dB}$ & $7.93 \%$ & $0.16 \mathrm{~dB}$ & $2.85 \%$ & $0.53 \mathrm{~dB}$ \\
\hline$T_{F_{3}}$ & 1.07 & 35.35 & 1.346 & 31.72 & 1.427 & 32.36 \\
\hline Improvements & $10.23 \%$ & $0.03 \mathrm{~dB}$ & $11.47 \%$ & $0.01 \mathrm{~dB}$ & $9.45 \%$ & $0.18 \mathrm{~dB}$ \\
\hline
\end{tabular}

Table 6: Performance improvement using the proposed method compared to the standard quantization table

\begin{tabular}{|c|c|c|c|c|c|c|}
\hline & \multicolumn{2}{|c|}{ Face } & \multicolumn{2}{c|}{ Body } & \multicolumn{2}{c|}{ Background } \\
\cline { 2 - 7 } & $\mathrm{bpp}$ & $\mathrm{dB}$ & $\mathrm{bpp}$ & $\mathrm{dB}$ & $\mathrm{bpp}$ & $\mathrm{dB}$ \\
\hline$T_{S}$ & 1.151 & 35.42 & 1.402 & 33.47 & 1.226 & 34.61 \\
\hline$T_{F_{1}}$ & 1.069 & 35.56 & 1.331 & 33.83 & 1.161 & 34.85 \\
\hline Improvements & $7.12 \%$ & $0.14 \mathrm{~dB}$ & $5.06 \%$ & $0.36 \mathrm{~dB}$ & $5.30 \%$ & $0.24 \mathrm{~dB}$ \\
\hline$T_{F_{2}}$ & 1.060 & 35.64 & 1.320 & 33.52 & 1.143 & 34.8 \\
\hline Improvements & $8.51 \%$ & $0.22 \mathrm{~dB}$ & $6.16 \%$ & $0.05 \mathrm{~dB}$ & $7.14 \%$ & $0.19 \mathrm{~dB}$ \\
\hline$T_{F_{3}}$ & 1.024 & 35.5 & 1.265 & 33.67 & 1.065 & 34.63 \\
\hline Improvements & $11.03 \%$ & $0.08 \mathrm{~dB}$ & $9.77 \%$ & $0.2 \mathrm{~dB}$ & $13.13 \%$ & $0.02 \mathrm{~dB}$ \\
\hline
\end{tabular}

\section{Conclusion}

In this paper, we presented a new JPEG quantization table design method for mobile images and the experimental results for the selected images. Based on the characteristics of mobile images, we proposed a new quantization table model. Also, considering the R-D cost function, the pre-emphasis factors and the bias factors were selected for different image groups. The experimental results showed the validity of the proposed method. Since the model is obtained from the standard quantization table in the proposed scheme, only the pre-emphasis factor and the bias factor need to be transmitted. The proposed scheme can be easily applied to the JPEG codec and can be utilized for the display of $240 \times 320$ images or other size images in mobile phones. 


\section{Acknowledgments}

This work was supported in part by the research program 2010 of Kookmin University, Korea and also supported in part by the Ministry of Knowledge Economy (MKE), Korea, under the Information Technology Research Center (ITRC) support program supervised by the Institute for Information Technology Advancement (IITA) under Grant IITA-2009-C1090-0904-0002.

\section{Bibliography}

[1] G. K. Wallace, The JPEG Still-Picture Compression Standard, Communications of the ACM, Vol. 34, No. 4, pp. 30-44, 1991.

[2] Independent JPEG Group, http://www.ijg.org.

[3] G.-M. Jeong, J.-H. Kang, Y.-S. Mun and D.-H. Jung, JPEG Quantization Table Design for Photos with Face in Wireless Handset, Lecture Notes in Computer Science, Vol. 3333, pp.681-688, 2004

[4] G.-M. Jeong, J.-D. Lee and D.-W. Kang, A JPEG Quantization Table for Mobile QVGA Images, The Journal of The Institute of Webcasting, Internet Television and Telecommunication (in Korean), Vol. 8, No. 1, pp.19-24, 2008

[5] SK Telecom, http://www.sktelecom.com

[6] M. Crouse and K. Ramchandran, Joint Thresholding and Quantizer Selection for Transform Image Coding: Entropy-Constrained Analysis and Applications to Baseline JPEG, IEEE Transactions on Image Processing, Vol. 6, No. 2, pp.285-297, 1997

Gu-Min Jeong received the B.S. and M.S. degrees from the Dept. of Control and Instrumentation Eng., Seoul National University, Seoul, Korea, in 1995 and 1997, respectively, and Ph.D. degree from School of Electrical Eng. and Computer Science, Seoul National University, Seoul, Korea in 2001. He was a Senior Engineer at NeoMtel, Korea from 2001-2004 and a Manager at SK Telecom, Korea from 2004-2005. Currently, he is an Associate Professor of School of Electrical Engineering, Kookmin University, Seoul, Korea. His research area includes wireless communication service, mobile multimedia, and embedded systems.

Jong-Duck LEE received the B.S. and M.S. degree from the School of Electrical Eng. Kookmin University, Seoul, Korea, in 2007 and 2009, respectively. Currently, he is working for LIG Nex1, Korea. His research area includes embedded systems and mobile multimedia.

Sang-Il Choi received his B.S. degree in the Division of Electronic Engineering from Sogang University in 2005, and received M.S. and Ph. D. degrees from School of Electrical Eng. and Computer Science, Seoul National University, Seoul, Korea, in 2007 and 2010, respectively. Currently, $\mathrm{He}$ is a post doctoral researcher in BK21 information technology in Seoul National University, Korea. His research interests include image processing, face recognition, feature extraction and their applications.

Dong-Wook Kang received the B.S., M.S., and Ph.D. degrees from the Dept. of Electronics Eng., Seoul National University, Seoul, Korea, in 1986, 1988, and 1995, respectively. He joined as a faculty member for the Dept. of Electrical Eng., Kookmin University, Seoul, Korea, in 1995 and now is a Professor of School of Electrical Eng., Kookmin University. He is a trustee of the Korean Society of Broadcasting Engineers. His research area includes video coding, multimedia signal processing and digital culture technology. 